\title{
Subglottic secretion suction for preventing ventilator-associated pneumonia: an updated meta-analysis and trial sequential analysis
}

Zhi Mao ${ }^{1 \dagger}$, Ling Gao ${ }^{2 \dagger}$, Guoqi Wang ${ }^{3 \dagger}$, Chao Liu', Yan Zhao ${ }^{1}$, Wanjie Gu${ }^{4}$, Hongjun Kang ${ }^{1}$ and Feihu Zhou ${ }^{1 *}$

\begin{abstract}
Background: Potential benefits of subglottic secretion suction for preventing ventilator-associated pneumonia (VAP) are not fully understood.

Methods: We searched Cochrane Central, PubMed, and EMBASE up to March 2016 to identify randomized controlled trials (RCTs) that compared subglottic secretion suction versus non-subglottic secretion suction in adults with mechanical ventilation. Meta-analysis was conducted using Revman 5.3, trial sequential analysis (TSA) 0.9 and STATA 12.0. The primary outcome was incidence of VAP. The Grading of Recommendations Assessment, Development, and Evaluation (GRADE) was used to evaluate the level of evidence.

Results: Twenty RCTs ( $N=3544)$ were identified. Subglottic secretion suction was associated with reduction of VAP incidence in four high quality trials (relative risk (RR) $0.54,95 \%$ confidence interval (Cl) $0.40-0.74 ; p<0.00001$ ) and in all trials ( $R R=0.55,95 \% \mathrm{Cl} 0.48-0.63 ; p<0.00001$ ). Sensitivity analyses did not show differences in the pooled results. Additionally, the results of the above-mentioned analyses were confirmed in TSA. GRADE level was high. Subglottic secretion suction significantly reduced incidence of early onset VAP, gram-positive or gram-negative bacteria causing VAP, and duration of mechanical ventilation. It delayed the time-to-onset of VAP. However, no significant differences in late onset VAP, intensive care unit (ICU) mortality, hospital mortality, or ICU length of stay were found.

Conclusions: Subglottic secretion suction decreased VAP incidence and duration of mechanical ventilation and delayed VAP onset. However, subglottic secretion suction did not reduce mortality and length of ICU stay. Subglottic secretion suction is recommended for preventing VAP and for reducing ventilation length, especially in the population at high risk of early onset VAP.
\end{abstract}

Trial registration: A protocol of this meta-analysis has been registered on PROSPERO (registration number: CRD42015015715); registered on 5 January 2015.

Keywords: Subglottic secretion suction, Ventilator-associated pneumonia, Meta-analysis, Trial sequential analysis

\footnotetext{
*Correspondence: feihuzhou301@126.com

${ }^{\dagger}$ Equal contributors

'Department of Critical Care Medicine, Chinese People's Liberation Army

General Hospital, 28 Fu-Xing Road, Beijing 100853, People's Republic of

China

Full list of author information is available at the end of the article
} 


\section{Background}

Ventilator-associated pneumonia (VAP) is a common clinical issue for patients receiving mechanical ventilation in the intensive care unit (ICU) [1]. The incidence of VAP accounts for $9 \%$ to $27 \%$ endotracheal intubated patients, whereas VAP has a mortality rate ranging from $25 \%$ to $50 \%$ [2-4]. VAP increased ICU and hospital length of stay, antibiotic consumption, and healthcare cost [5-8]. The primary mechanism of VAP is the microaspiration of the accumulated secretions around the endotracheal tube cuff. Preventive measures for microaspiration and VAP include continuous control of tracheal cuff pressure, conical cuff shape, and subglottic secretion suctioning (SSS).

SSS has been recommended in several guidelines to prevent the occurrence of VAP $[9,10]$. Previous meta-analyses reported that SSS was associated with a lower rate of VAP [11-16]. However, many ICUs did not use SSS as a part of the VAP bundle [17]. Although about $55 \%$ of hospitals in the US routinely used SSS in 2013 [18], European consensus did not recommend SSS for VAP prevention [19]. The prevention of VAP by SSS is not fully proven based on current evidence. The effect of SSS on early- or late-onset VAP, duration of mechanical ventilation, ICU length of stay, and mortality is controversial [16]. The need for future randomized controlled trials (RCTs) is still advocated $[15,16]$. Six RCTs [17, 20-24] involving new evidence on this topic have been published recently.

Therefore, we conducted an updated meta-analysis of RCTs to evaluate the effect of SSS on VAP prevention. Trial sequential analysis (TSA) was used to determine whether the currently evidence was robust and conclusive.

\section{Methods}

The Preferred Reporting Items for Systematic Reviews and Meta-Analyses (PRISMA) were used to report this systematic review and meta-analysis [25]. A protocol for this meta-analysis has been registered on PROSPERO (http://www.crd.york.ac.uk/prospero), and the registration number is CRD42015015715.

\section{Eligibility criteria}

The inclusion criteria were as follows: 1) patients received mechanical ventilator ( $\geq 48 \mathrm{~h}) ; 2$ ) intervention was SSS regardless of continuous or intermittent SSS; 3) the control was the non-subglottic secretion suctioning group; 4) the incidence of VAP was reported; and 5) the study design was RCT. The exclusion criteria were patients younger than 18 years and repeated data.

\section{Search strategy}

A literature search of Cochrane Central (March 2016), PubMed (1950 to March 2016), and EMBASE (1980 to March 2016) was undertaken to identify trials according to Cochrane Collaboration methodology. The search terms used were "ventilator-associated pneumonia" with "subglottic secretion" or "subglottic drainage" or "subglottic suctioning" or "glottic" and "randomized" or "randomised". No language restriction was applied. We also hand-searched the reference lists of review articles and conference proceedings.

\section{Study selection}

Two independent reviewers (ZM and LG) conducted the study selection. Disagreements between the two reviewers were resolved in meetings or adjudicated by a third reviewer $(\mathrm{GW})$.

\section{Data extraction}

For each included study, data extraction was performed independently by two reviewers using a standard form. The following data on study characteristics were collected: year of publication, the study type, number of patients, patient characteristics, method of SSS, definition of VAP, incidence of VAP, length of ICU stay, duration of mechanical ventilation (MV), mortality, airway secretion bacteria detection, and details of the outcomes. The quality of included studies was assessed by the Cochrane risk of bias tool [26]. Trials with low risk of bias in all items were evaluated as high-quality studies.

\section{Grading the quality of evidence}

Grading of Recommendations Assessment, Development, and Evaluation (GRADE) methodology was used to assess the quality of evidence classified as high, moderate, low, or very low. The judgments were based on risk of bias, inconsistency, indirectness, imprecision, and publication bias. GRADE Pro-version 3.6 software was used.

\section{Statistical analysis}

Dichotomous variables were presented as relative risks (RRs), and continuous variables were presented as mean difference (MD), both with $95 \%$ confidence intervals (CIs). Heterogeneity across studies was evaluated by visual inspection of the forest plot, along with quantification using the $I^{2}$ statistic. An $I^{2}>50 \%$ was considered as significant heterogeneity. A fixed-effects model was used in the meta-analysis unless significant heterogeneity among studies was present. The random-effects model of DerSimonian and Laird [27] was used regardless of heterogeneity. Statistical analysis was performed with Review Manager 5.1 software (Cochrane Collaboration, Oxford, UK) for outcome measures. A $p$ value $<0.05$ was considered statistically significant. We assessed publication bias by visually inspecting a funnel plot. The Begg and Egger tests were also conducted using STATA 12.0.

For the primary result, predefined subgroup analyses were performed as the high-quality subgroup, and the 
primary result from high-quality trials was emphasized. Sensitivity analysis for primary results were conducted by excluding trials with multiple manipulations, continuous versus intermittent suction, appropriate randomization, allocation concealment, assessment blinding, and participants numbering more than 100 . We also performed sensitivity analyses using an invasive diagnosis of VAP in all outcomes.

The second outcomes were incidence of early- or lateonset VAP, gram-positive or gram-negative bacteria causing VAP, ICU or hospital mortality, time-to-onset of VAP, duration of mechanical ventilation, ICU or hospital length of stays, and incidence of tracheotomy or reintubation.

\section{Trial sequential analysis}

TSA, which is similar to an interim trial analysis in a single trial, was conducted to obtain the primary result. Cumulative meta-analysis that is updated with new studies may result in false positive results (type I error) because of an increased risk of random error from sparse data and repeated significance testing [28]. TSA can control the $p$ value and widen the confidence intervals [29]. TSA combined concepts and rationale as follows: an estimation of the required information size and trial sequential monitoring boundaries. If the cumulative $\mathrm{Z}$ curve enters the futility area or crosses the trial sequential monitoring boundary, the anticipated intervention effect may reach a sufficient level of evidence, and further trials will not be necessary. If the $\mathrm{Z}$ curve does not cross any of the boundaries or reach the required information size, evidence is insufficient for drawing a conclusion.

We calculated the required information size based on a relative risk reduction of $20 \%$ in incidence of VAP. The type I error $(\alpha)$ and power $(1-\beta)$ were set as 0.05 and 0.80 , respectively. The control event rates were calculated from the non-subglottic secretion suctioning group. The TSA was conducted with the use of TSA version 0.9 beta software (http://www.ctu.dk/tsa).

\section{Results}

\section{Trial selection}

A total of 11,756 potentially relevant articles were used. We excluded duplicate studies, non-relevant topic articles, non-RCTs, and non-suitable intervention studies. Twenty studies reported that 3544 patients were included in this meta-analysis (Fig. 1) [17, 20-24, 30-43].

\section{Trials characteristics}

The main characteristics of the selected studies are summarized in Table 1 . These studies were reported between 1992 and 2016, and a total of 3544 patients were included. Fourteen studies were published in English $[17,20,21,23,24,30-32,34-36,38,39,42]$, five in

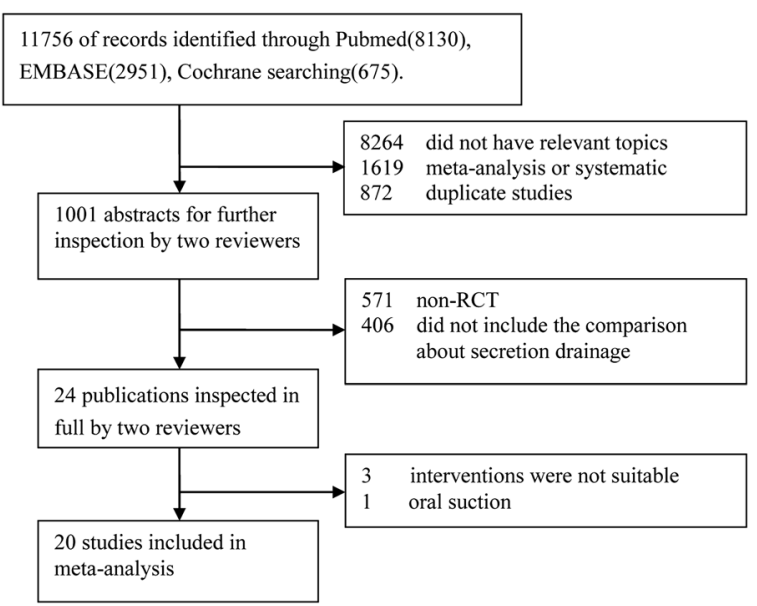

Fig. 1 Flow chart of the trial selection. $R C T$ randomized controlled trial

Chinese [22, 33, 37, 40, 41], and one in Persian [43]. One abstract was included [24].

\section{Risk of bias assessment}

Risk of bias is summarized in Fig. 2. Twelve studies reported and adequate randomized sequence generated $[17,20-22,34-36,38-42]$, five studies reported appropriate allocation concealment $[17,20,34,39,42]$, and seven studies reported blinding of outcome assessments $[17,23,31,34,35,38,42]$. Four studies were highquality studies with low risk of bias in all items (Fig. 2) $[17,20,34,42]$.

\section{Primary outcome: incidence of VAP}

Four high-quality studies with 901 participants were included in the analysis of VAP incidence (Fig. 3) $[17,20,34,42]$, thereby suggesting an $R R$ of 0.54 $(95 \%$ CI $0.40-0.74 ; p<0.00001 ; p$ for heterogeneity $=$ $\left.0.39, I^{2}=0 \%\right)$ for SSS versus non-SSS. Absolute risk reduction (ARR) was 0.0953. The number needed to treat (NNT) was 10.49. TSA showed that the cumulative $\mathrm{Z}$ curves crossed both the conventional boundary and the trial sequential monitoring boundary. Thus, further trials were unlikely to change the conclusion (Fig. 3, Table 2).

Twenty studies totalling 3544 patients provided data on incidence of VAP despite a risk of bias. Overall, subglottic secretion suction significantly prevented the incidence of VAP $(\mathrm{RR}=0.55,95 \%$ CI $0.48-0.63 ; p<0.00001 ; p$ for heterogeneity $=0.85, I^{2}=0 \%$ ) (Fig. 1 ). TSA showed that the cumulative $\mathrm{Z}$ curves crossed both the conventional and the trial sequential monitoring boundaries and reached the significant area, which led us to draw a conclusion. Thus, it is unlikely that further trials will change the conclusion and are not necessary (Fig. 4).

Sensitivity analyses were performed to evaluate only including trials using invasive diagnosis of VAP, the 
Table 1 Characteristics of included studies

\begin{tabular}{|c|c|c|c|c|c|c|c|c|}
\hline Study & $\begin{array}{l}\text { Population } \\
\text { (n) }\end{array}$ & Settings & $\begin{array}{l}\text { Definition } \\
\text { of VAP }\end{array}$ & Inclusion criteria & Exclusion criteria & $\begin{array}{l}\text { Method } \\
\text { of SSS }\end{array}$ & Antibiotic use & $\begin{array}{l}\text { Evaluation unit } \\
\text { of antibiotic } \\
\text { consumption }\end{array}$ \\
\hline $\begin{array}{l}\text { Mahul, } \\
1992[30]\end{array}$ & 145 & Medical-surgical ICU & $\begin{array}{l}\text { Positive bronchoalveolar } \\
\text { lavage culture required }\end{array}$ & $\begin{array}{l}\text { Expected duration } \\
\text { of } \mathrm{MV}>3 \text { days }\end{array}$ & $\begin{array}{l}\text { Gastrointestinal bleeding, } \\
\text { tracheostomy, risk of } \\
\text { reintubation, intubated } \\
\text { before ICU }\end{array}$ & Intermittent & $\begin{array}{l}\text { Antibiotic use at } \\
\text { randomization not } \\
\text { reported }\end{array}$ & NA \\
\hline $\begin{array}{l}\text { Valles, } \\
1995 \text { [31] }\end{array}$ & 153 & Medical-surgical ICU & $\begin{array}{l}\text { Clinical features confirmed } \\
\text { with bronchoscopically } \\
\text { obtained cultures }\end{array}$ & $\begin{array}{l}\text { Expected duration } \\
\text { of } \mathrm{MV}>3 \text { days }\end{array}$ & $\begin{array}{l}\text { Intubated before arriving } \\
\text { at the emergency } \\
\text { department or ICU; } \\
\text { tracheostomy }\end{array}$ & Continuous & $\begin{array}{l}\text { Patients receiving } \\
\text { antibiotics at time of } \\
\text { randomization: } 64 \% \\
\text { and } 58 \% \text {, intervention } \\
\text { and control group } \\
\text { respectively }\end{array}$ & NA \\
\hline $\begin{array}{l}\text { Kollef, } \\
1999 \text { [32] }\end{array}$ & 343 & Cardiothoracic ICU & $\begin{array}{l}\text { Clinical features, positive } \\
\text { tracheal, blood, or pleural } \\
\text { cultures; radiographic } \\
\text { abscess, or positive } \\
\text { histology }\end{array}$ & $\begin{array}{l}\text { Need for MV after } \\
\text { cardiac surgery }\end{array}$ & $\begin{array}{l}\text { Intubated before ICU; } \\
\text { transfer from outside } \\
\text { hospital }\end{array}$ & Continuous & $\begin{array}{l}\text { Antibiotics were given } \\
\text { to } 99 \% \text { and } 98 \% \text { of } \\
\text { intervention and control } \\
\text { patients, respectively }\end{array}$ & NA \\
\hline $\begin{array}{l}\text { Bo, } \\
2000[33]\end{array}$ & 68 & Surgical ICU & $\begin{array}{l}\text { Clinical features or positive } \\
\text { blood/pleural cultures }\end{array}$ & $\begin{array}{l}\text { Expected duration } \\
\text { of } \mathrm{MV}>72 \mathrm{~h}\end{array}$ & $\begin{array}{l}\text { Intubated outside } \\
\text { hospital; high-risk surgery } \\
\text { or trauma; pre-existing } \\
\text { infection }\end{array}$ & Continuous & $\begin{array}{l}\text { Antibiotics were used } \\
\text { in } 29 \% \text { of intervention } \\
\text { group and } 36 \% \text { of } \\
\text { control group }\end{array}$ & NA \\
\hline $\begin{array}{l}\text { Smulders, } \\
2002[34]\end{array}$ & 150 & Medical-surgical ICU & $\begin{array}{l}\text { Clinical features or positive } \\
\text { blood/pleural cultures }\end{array}$ & $\begin{array}{l}\text { Expected duration } \\
\text { of } \mathrm{MV}>72 \mathrm{~h}\end{array}$ & $\begin{array}{l}\text { Patients expected to } \\
\text { require }>72 \mathrm{~h} \mathrm{MV}\end{array}$ & Intermittent & $\begin{array}{l}48 \% \text { of intervention } \\
\text { group and } 51 \% \text { of } \\
\text { control group were } \\
\text { receiving antibiotics }\end{array}$ & NA \\
\hline $\begin{array}{l}\text { Girou, } \\
2004[35]\end{array}$ & 18 & NA & $\begin{array}{l}\text { Clinical features and } \\
\text { significant quantitative } \\
\text { culture of aspiration }\end{array}$ & $\begin{array}{l}\text { Expected duration } \\
\text { of } \mathrm{MV}>5 \text { days }\end{array}$ & NA & Continuous & $\begin{array}{l}\text { Prior antibiotic therapy: } \\
1 \text { patient in suctioning } \\
\text { group and } 4 \text { patients } \\
\text { in control group }\end{array}$ & NA \\
\hline $\begin{array}{l}\text { Liu SH, } \\
2006[37]\end{array}$ & 98 & Respiratory ICU & $\begin{array}{l}\text { MV }>48 \text { h, the chest X-ray } \\
\text { showed pulmonary new } \\
\text { or progressive infiltration } \\
\text { lesions, and excluding } \\
\text { atelectasis, pulmonary } \\
\text { edema, and pleural effusion }\end{array}$ & $\begin{array}{l}\text { Age older than } \\
60 \text { years, expected } \\
M V>48 \mathrm{~h}\end{array}$ & NA & Intermittent & NA & NA \\
\hline $\begin{array}{l}\text { Liu QH, } \\
2006[36]\end{array}$ & 86 & NA & $\begin{array}{l}\text { Received MV for }>48 \mathrm{~h} \text {, } \\
\text { clinical features and } \\
\text { culture of endotracheal } \\
\text { aspirate; reduction } \\
\text { of oxygen }\end{array}$ & $\begin{array}{l}\text { Age older than } \\
60 \text { years, expected } \\
M V>48 \mathrm{~h}\end{array}$ & $\begin{array}{l}\text { 1) expected death within } \\
48 \mathrm{~h} ; 2) \text { expected weaning } \\
\text { within } 48 \mathrm{~h} ; 3 \text { ) existing } \\
\text { lung infection when } \\
\text { MV beginning }\end{array}$ & Continuous & NA & NA \\
\hline
\end{tabular}


Table 1 Characteristics of included studies (Continued)

\begin{tabular}{|c|c|c|c|c|c|c|c|c|}
\hline $\begin{array}{l}\text { Lorente, } \\
2007[38]\end{array}$ & 280 & NA & $\begin{array}{l}\text { Clinical features and } \\
\text { significant quantitative } \\
\text { culture via ETT aspiration }\end{array}$ & $\begin{array}{l}\text { Expected MV } \\
>24 \mathrm{~h}\end{array}$ & $\begin{array}{l}\text { Age less than } 18 \text { years, } \\
\text { pregnancy, infection with } \\
\text { human immunodeficiency } \\
\text { virus, blood leukocyte count } \\
\text { less than } 1000 \text { cells/mm3, } \\
\text { solid or hematological tumor, } \\
\text { and/or immunosuppressive } \\
\text { therapy }\end{array}$ & Continuous & $\begin{array}{l}83.6 \% \text { of ETT-PUC-SSD } \\
\text { group and } 85 \% \text { of ETT-C } \\
\text { group were receiving } \\
\text { antibiotics after } \\
\text { cardiothoracic surgery }\end{array}$ & NA \\
\hline $\begin{array}{l}\text { Bouza, } \\
2008[39]\end{array}$ & 704 & Cardiothoracic ICU & $\begin{array}{l}\text { Received MV for }>48 \mathrm{~h} \text {, } \\
\text { clinical features and } \\
\text { culture of endotracheal } \\
\text { aspirate; reduction } \\
\text { of oxygen }\end{array}$ & Major heart surgery & NA & Continuous & $\begin{array}{l}\text { Both group respectively } \\
\text { received antibiotics } \\
\text { before surgery and } \\
\text { every } 8 \mathrm{~h} \text { thereafter for } \\
\text { a total of three doses } \\
\text { in the ICU }\end{array}$ & $\begin{array}{l}\text { Daily defined } \\
\text { doses }\end{array}$ \\
\hline $\begin{array}{l}\text { Yang, } \\
2008[40]\end{array}$ & 91 & Medical-surgical ICU & $\begin{array}{l}\text { Clinical features and } \\
\text { culture of endotracheal } \\
\text { aspirate }\end{array}$ & $M V>48 h$ & Intubated before ICU & Continuous & NA & NA \\
\hline $\begin{array}{l}\text { Zheng, } \\
2008 \text { [41] }\end{array}$ & 61 & Medical-surgical ICU & NA & $M V>48 h$ & NA & Continuous & NA & NA \\
\hline $\begin{array}{l}\text { Lacherade, } \\
2010 \text { [42] }\end{array}$ & 333 & $\begin{array}{l}\text { Multicenter, } \\
\text { medical-surgical } \\
\text { ICU }\end{array}$ & $\begin{array}{l}\text { Quantitative culture } \\
\text { of protected telescoping } \\
\text { catheter sample or } \\
\text { bronchoalveolar lavage } \\
\text { fluid following } \\
\text { clinical suspicion }\end{array}$ & $\begin{array}{l}\text { Expected MV } \\
>48 \mathrm{~h}\end{array}$ & $\begin{array}{l}\text { Intubated before ICU; } \\
\text { tracheostomy; psychotropic } \\
\text { drug overdose; acute } \\
\text { drunkenness; cardiac arrest }\end{array}$ & Intermittent & $\begin{array}{l}\text { Antibiotics therapy was } \\
\text { used in } 94 \% \text { and } 92 \% \\
\text { of intervention and } \\
\text { control groups } \\
\text { respectively }\end{array}$ & NA \\
\hline $\begin{array}{l}\text { Seyfi, } \\
2013 \text { [43] }\end{array}$ & 80 & $\begin{array}{l}\text { ICU of Hazrat Rasool } \\
\text { Akram Hospital of } \\
\text { Tehran, Iran. }\end{array}$ & NA & NA & NA & Intermittent & NA & NA \\
\hline $\begin{array}{l}\text { Safdari, } \\
2014 \text { [23] }\end{array}$ & 76 & $\begin{array}{l}\text { In four ICUs of } \\
\text { Educational } \\
\text { Hospital in Isfahan, } \\
\text { Iran }\end{array}$ & NA & $\begin{array}{l}\text { Intubated with } \\
\text { a conventional endotracheal } \\
\text { tube and connected to } \\
\text { ventilators for more than } \\
48 \mathrm{~h}\end{array}$ & $\begin{array}{l}\text { Patients who were admitted } \\
\text { to the ICUs with tracheostomy } \\
\text { or likely to die in the next } \\
48 \mathrm{~h} \text { or admitted to these } \\
\text { units for treatment of } \\
\text { pneumonia or with lung } \\
\text { complications like fibrosis } \\
\text { or cancer }\end{array}$ & $\begin{array}{l}\text { Intermittent } \\
\text { performed every } \\
3 \mathrm{~h}\end{array}$ & NA & NA \\
\hline $\begin{array}{l}\text { Koker, } \\
2014[24]\end{array}$ & 51 & In a 14-bed ICU & NA & $\begin{array}{l}\text { Requiring prolonged MV } \\
\text { for more than } 48 \mathrm{~h}\end{array}$ & NA & Continuous & NA & NA \\
\hline $\begin{array}{l}\text { Tao, } \\
2014 \text { [22] }\end{array}$ & 149 & NA & $\begin{array}{l}\text { Received MV for }>48 \mathrm{~h} \text {, } \\
\text { clinical features and } \\
\text { culture of endotracheal } \\
\text { aspirate; reduction } \\
\text { of oxygen }\end{array}$ & $\begin{array}{l}\text { Expected MV }>48 \text { h with } \\
\text { APACHE score } 20-30\end{array}$ & $\begin{array}{l}\text { Existing lung infection } \\
\text { when MV beginning }\end{array}$ & $\begin{array}{l}\text { Continuous or } \\
\text { intermittent every } \\
4 \mathrm{~h}\end{array}$ & NA & NA \\
\hline
\end{tabular}


Table 1 Characteristics of included studies (Continued)

\begin{tabular}{|c|c|c|c|c|c|c|c|c|}
\hline $\begin{array}{l}\text { Damas, } \\
2015[17]\end{array}$ & 352 & $\begin{array}{l}\text { All ICUs of a tertiary } \\
\text { hospital }\end{array}$ & $\begin{array}{l}\text { Clinical features and } \\
\text { culture of endotracheal } \\
\text { aspirate }\end{array}$ & $\begin{array}{l}\text { Age over } 18 \text { years, } \\
\text { intubation with a } \\
\text { Teleflex Isis }{ }^{T M} \\
\text { endotracheal tube (TIET) } \\
\text { allowing subglottic } \\
\text { secretion suctioning }\end{array}$ & $\begin{array}{l}\text { Patient participating in } \\
\text { another study or having } \\
\text { already participated in } \\
\text { this study }\end{array}$ & NA & NA & $\begin{array}{l}\text { The numberof } \\
\text { antibiotic days }\end{array}$ \\
\hline $\begin{array}{l}\text { Gopal, } \\
2015 \text { [21] }\end{array}$ & 240 & $\begin{array}{l}\text { Cardiothoracic } \\
\text { surgery }\end{array}$ & $\begin{array}{l}\text { Europe Infection Control } \\
\text { through Surveillance } \\
\text { definition }\end{array}$ & $\begin{array}{l}\text { Age over } 70 \text { years and/or } \\
\text { left ventricular ejection } \\
\text { fraction }<50 \% \text { and } \\
\text { cardiac surgery }\end{array}$ & NA & $\begin{array}{l}\text { Intermittent every } \\
6 \mathrm{~h}\end{array}$ & NA & NA \\
\hline $\begin{array}{l}\text { Deem, } \\
2016 \text { [20] }\end{array}$ & 66 & $\begin{array}{l}\text { All ICUs at } \\
\text { Harborview Medical } \\
\text { Center }\end{array}$ & $\begin{array}{l}\text { Criteria of Center for } \\
\text { Disease Control }\end{array}$ & $\begin{array}{l}\text { Age over } 18 \text { years with } \\
\text { intubation and needed } \\
\text { critical care }\end{array}$ & $\begin{array}{l}\text { 1) Out-of-hospital cardiac } \\
\text { arrest; 2) non-study } \\
\text { designated intubation } \\
\text { devices; 3) airway } \\
\text { management other than } \\
\text { orotracheal intubation; } \\
\text { 4) Federally protected } \\
\text { populations, pregnant } \\
\text { women, and prisoners }\end{array}$ & Continuous & NA & NA \\
\hline
\end{tabular}

APACHE Acute Physiology and Chronic Health Evaluation, ETT-C conventional endotracheal tube, ETT-PUC-SSD endotracheal tube with polyurethane cuff and subglottic secretion drainage, ICU intensive care unit,

$M V$ mechanical ventilation, NA not available, SSS subglottic secretion suctioning, VAP ventilator-associated pneumonia 


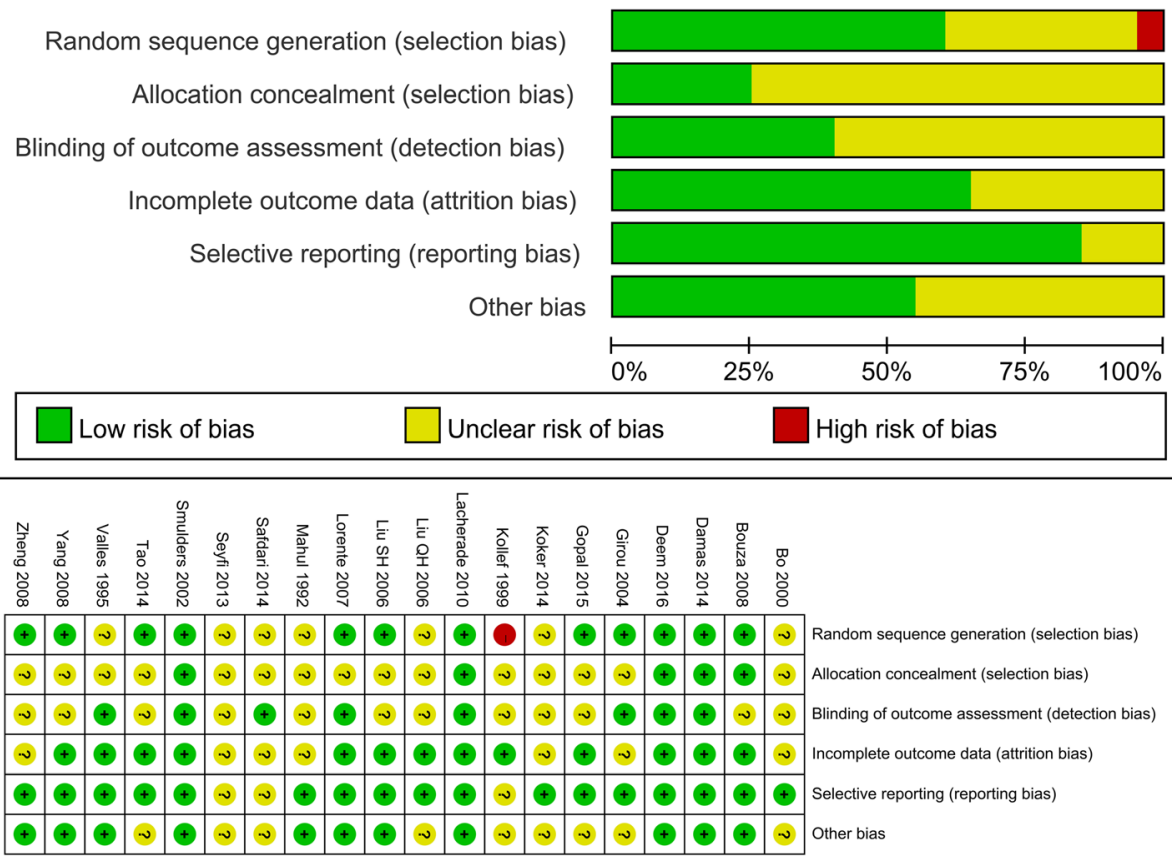

Fig. 2 Risk of bias table

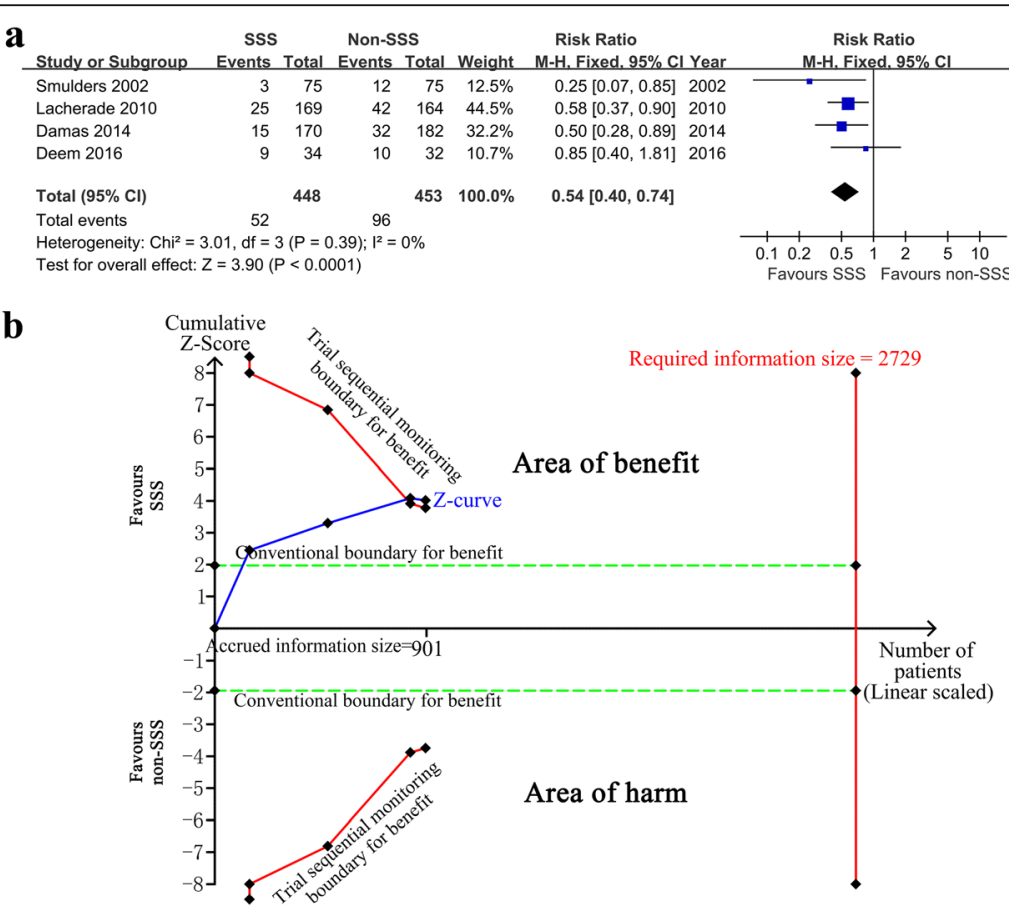

Fig. 3 Effect of subglottic secretion suction on preventing ventilator-associated pneumonia in four high-quality trials. a Forest plot comparing subglottic secretion suction (SSS) and non-SSS on incidence of ventilator-associated pneumonia. $\mathbf{b}$ Trial sequential analysis for incidence of ventilator-associated pneumonia with control arm event proportion of $21.2 \%$, relative risk reduction of $20 \%$, a of $5 \%$ (two sided), $\beta$ of $20 \%$, and $P^{2}=0 \%$. The required information size was calculated as 2729 . Z curve has across-trial sequential monitoring boundary for benefit. Risk of bias: A random sequence generation (selection bias), B allocation concealment (selection bias), $C$ blinding of outcome assessment (detection bias), $D$ incomplete outcome data (attrition bias), E selective reporting (reporting bias), $F$ other bias; Cl confidence interval, $M$ - $H$ Mantel-Haenszel 
Table 2 The GRADE evidence quality for primary outcome, sensitivity analysis, and secondary outcomes

\begin{tabular}{|c|c|c|c|c|c|c|c|}
\hline Categaries & Outcomes & Number of studies & $\begin{array}{l}\text { Risk ratio or mean } \\
\text { difference }(95 \% \mathrm{Cl})\end{array}$ & $P$ & $P$ for heterogeneity & $1^{2}(\%)$ & Quality \\
\hline \multirow[t]{10}{*}{ Primary outcome Sensitivity analysis } & VAP high-quality trials & $4[17,20,34,42]$ & $0.54(0.40,0.74)$ & $<0.00001$ & 0.39 & 0 & Moderate $^{c}$ \\
\hline & VAP total trials & $20[17,20-24,30-43]$ & $0.55(0.48,0.63)$ & $<0.00001$ & 0.85 & 0 & $\mathrm{High}^{\mathrm{a}}$ \\
\hline & VAP invasive diagnosis & $13[17,20,22,30,31,33,35-40,42]$ & $0.55(0.47,0.65)$ & $<0.00001$ & 0.60 & 0 & $\mathrm{High}^{\mathrm{a}}$ \\
\hline & $\begin{array}{l}\text { VAP excluding trials with multiple } \\
\text { manipulations }\end{array}$ & $16[17,22-24,30-34,36-43]$ & $0.55(0.48,0.65)$ & $<0.00001$ & 0.97 & 0 & $\mathrm{High}^{\mathrm{a}}$ \\
\hline & VAP randomization & $12[17,20-22,34,35,37-42]$ & $0.56(0.47,0.66)$ & $<0.00001$ & 0.37 & 7 & $\mathrm{High}^{\mathrm{a}}$ \\
\hline & VAP allocation concealment & $5[17,20,34,39,42]$ & $0.56(0.42,0.74)$ & $<0.00001$ & 0.53 & 0 & Moderate ${ }^{c}$ \\
\hline & VAP assessment blinded & $8[17,20,23,31,34,35,38,42]$ & $0.53(0.42,0.66)$ & $<0.00001$ & 0.34 & 11 & Moderate $^{c}$ \\
\hline & VAP participants more than 100 & $10[17,21,22,30-32,34,38,39,42]$ & $0.54(0.45,0.65)$ & $<0.00001$ & 0.55 & 0 & $\mathrm{High}^{\mathrm{a}}$ \\
\hline & VAP intermittent SSS & $9[21-23,30,34,37,38,42,43]$ & $0.52(0.43,0.64)$ & $<0.00001$ & 0.25 & 22 & High $^{a}$ \\
\hline & VAP continuous SSS & $11[20,22,24,31-33,35,36,39-41]$ & $0.61(0.5,0.73)$ & $<0.00001$ & 0.91 & 0 & $\mathrm{High}^{\mathrm{a}}$ \\
\hline \multirow[t]{12}{*}{ Secondary outcomes } & Early-onset VAP & $9[22-24,33,36-38,42,43]$ & $0.34(0.25,0.47)$ & $<0.00001$ & 0.84 & 0 & Moderate ${ }^{c}$ \\
\hline & Late-onset VAP & $5[22,33,36,38,42]$ & $0.80(0.62,1.02)$ & 0.07 & 0.17 & 35 & Moderate \\
\hline & Gram-negative bacteria & $6[31,32,38-40,42]$ & $0.58(0.43,0.77)$ & 0.0002 & 0.69 & 0 & Moderate $^{c}$ \\
\hline & Gram-positive bacteria & $5[31,33,38,40,42]$ & $0.32(0.17,0.61)$ & 0.006 & 0.61 & 0 & Low $^{\mathrm{b}}$ \\
\hline & ICU mortality & $8[17,20,30,31,38,39,41,42]$ & $0.98(0.85,1.13)$ & 0.77 & 0.88 & 0 & High \\
\hline & Hospital mortality & $7[17,21,32,34,37,40,42]$ & $0.92(0.80,1.05)$ & 0.20 & 0.82 & 0 & High \\
\hline & Time-to-onset of VAPg & $7[30-33,37,40,41]$ & $3.92(2.56,5.27)$ & $<0.00001$ & $<0.00001$ & 92 & Moderate $^{\mathrm{d}}$ \\
\hline & Duration of $\mathrm{MV}^{\mathrm{g}}$ & $6[20,32,34,37,38,41]$ & $-1.17(-2.28,-0.06)$ & 0.006 & 0.06 & 54 & Moderate $^{\mathrm{d}}$ \\
\hline & ICU length of stay ${ }^{9}$ & $4[32,34,38,41] 37$ & $-1.64(-3.95,0.66)$ & 0.16 & 0.001 & 81 & Moderate $^{\mathrm{d}}$ \\
\hline & Hospital length of stay & $3[32,34]$ & $-1.44(-3.93,1.04)$ & 0.25 & 0.99 & 0 & High \\
\hline & Reintubation & $4[20,32,39,42]$ & $0.77(0.45,1.32)$ & 0.34 & 0.19 & 38 & Low $^{b}$ \\
\hline & Tracheotomy ${ }^{g}$ & $3[32,38,42]$ & $1.14(0.75,1.72)$ & 0.55 & 0.79 & 54 & Low $^{\mathrm{b}}$ \\
\hline
\end{tabular}

CI confidence interval, GRADE Grading of Recommendations Assessment, Development, and Evaluation, ICU intensive care unit, $M V$ mechanical ventilation, NA not available, SSS subglottic secretion suctioning, VAP ventilator-associated pneumonia

aTotal number of events is more than 300

${ }^{\mathrm{b}}$ Total number of events is less than 100

'Total number of events is less than 300

$\mathrm{d}^{2}>50 \%$

eThe total number of patients is relatively small $(<500)$

${ }^{\mathrm{f}}$ The total number of patients is very small (100)

${ }^{9}$ Random-effect model 


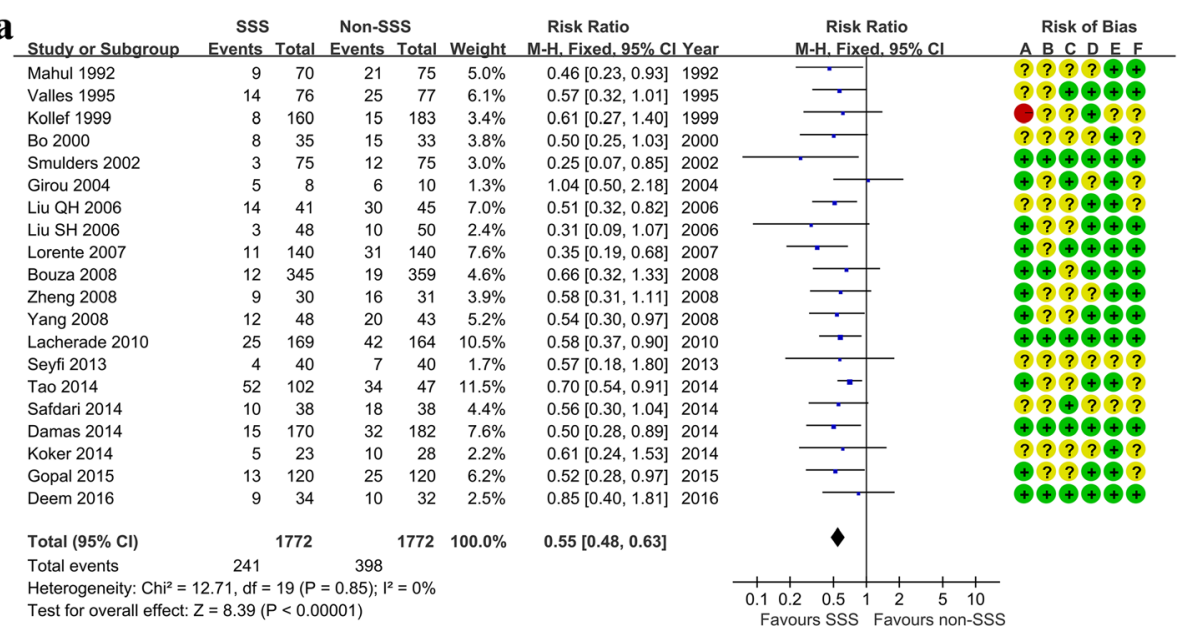

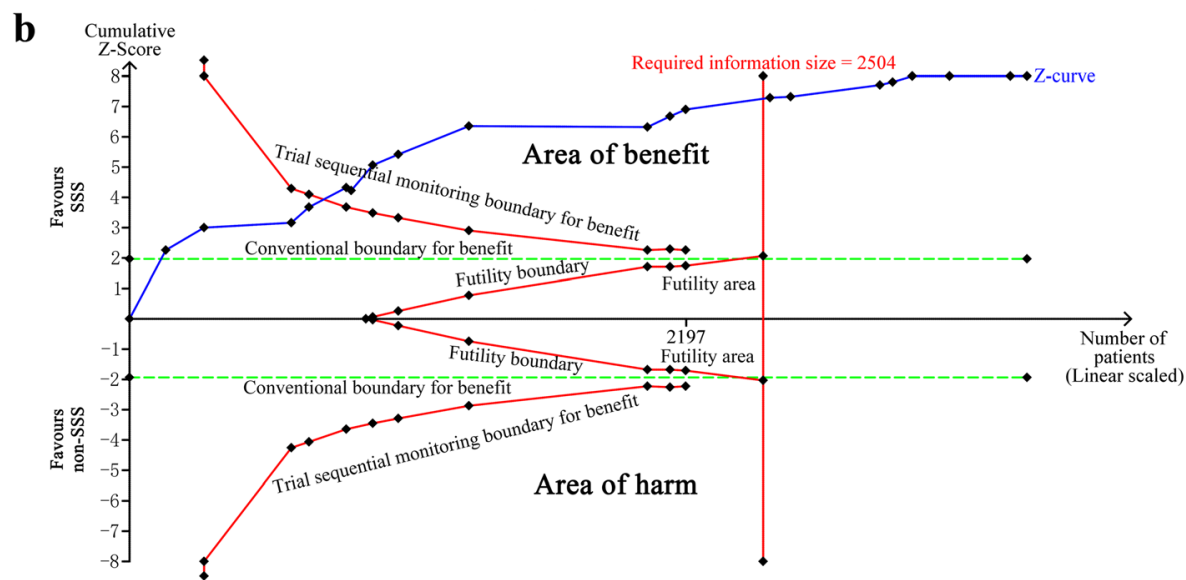

Fig. 4 Effect of subglottic secretion suction on preventing ventilator-associated pneumonia in all included trials. a Forest plot comparing subglottic secretion suction (SSS) and non-SSS on incidence of ventilator-associated pneumonia. $\mathbf{b}$ Trial sequential analysis for incidence of ventilator-associated pneumonia with control arm event proportion of $22.5 \%$, relative risk reduction of $20 \%$, a of $5 \%$ (two sided), $\beta$ of $20 \%$, and $P=0 \%$. The required information size was calculated as 2504 . Z curve has across-trial sequential monitoring boundary for benefit and required information size. Risk of bias: A random sequence generation (selection bias), $B$ allocation concealment (selection bias), C blinding of outcome assessment (detection bias), $D$ incomplete outcome data (attrition bias), E selective reporting (reporting bias), F other bias; Cl confidence interval, $\mathrm{M}-\mathrm{H}$ Mantel-Haenszel

influence of the intervention types, risk of bias, and sample size on the combined estimates (Table 2). Some confounding interventions, such as polyurethane cuff $[20,38]$, continuous control of cuff pressure [21], and semi-recumbent position [35] existed in some included trials. The sensitivity analysis excluded these trials, which used other confounding interventions [20, 21, 35, 38]. The result of sensitivity analysis was stable (Table 2). Additionally, TSA of sensitivity analyses were conducted to confirm the significant results (Fig. 5).

\section{Secondary outcomes}

SSS was significantly associated with a reduction in the incidence of early-onset VAP, gram-positive or gramnegative bacteria causing VAP, and duration of mechanical ventilation. SSS also delayed the time-to-onset of VAP. However, no significant difference was detected between
SSS and non-SSS in terms of late-onset VAP, ICU mortality, hospital mortality, ICU length of stay, incidence of tracheotomy, or reintubation (Table 2). Sensitivity analyses using an invasive diagnosis of VAP in all secondary outcomes did not show additional significant results (Additional file 1: Table S1).

\section{GRADE}

The GRADE level of evidence is high for VAP incidence, hospital mortality, and hospital length of stay, but moderate for early-onset VAP, gram-negative bacteria causing VAP, ICU mortality, time to achieve VAP, and duration of mechanical ventilation and ICU stay. Table 2 shows the GRADE evidence profiles. The main reasons for a deceasing level were inconsistency and limited sample size. 


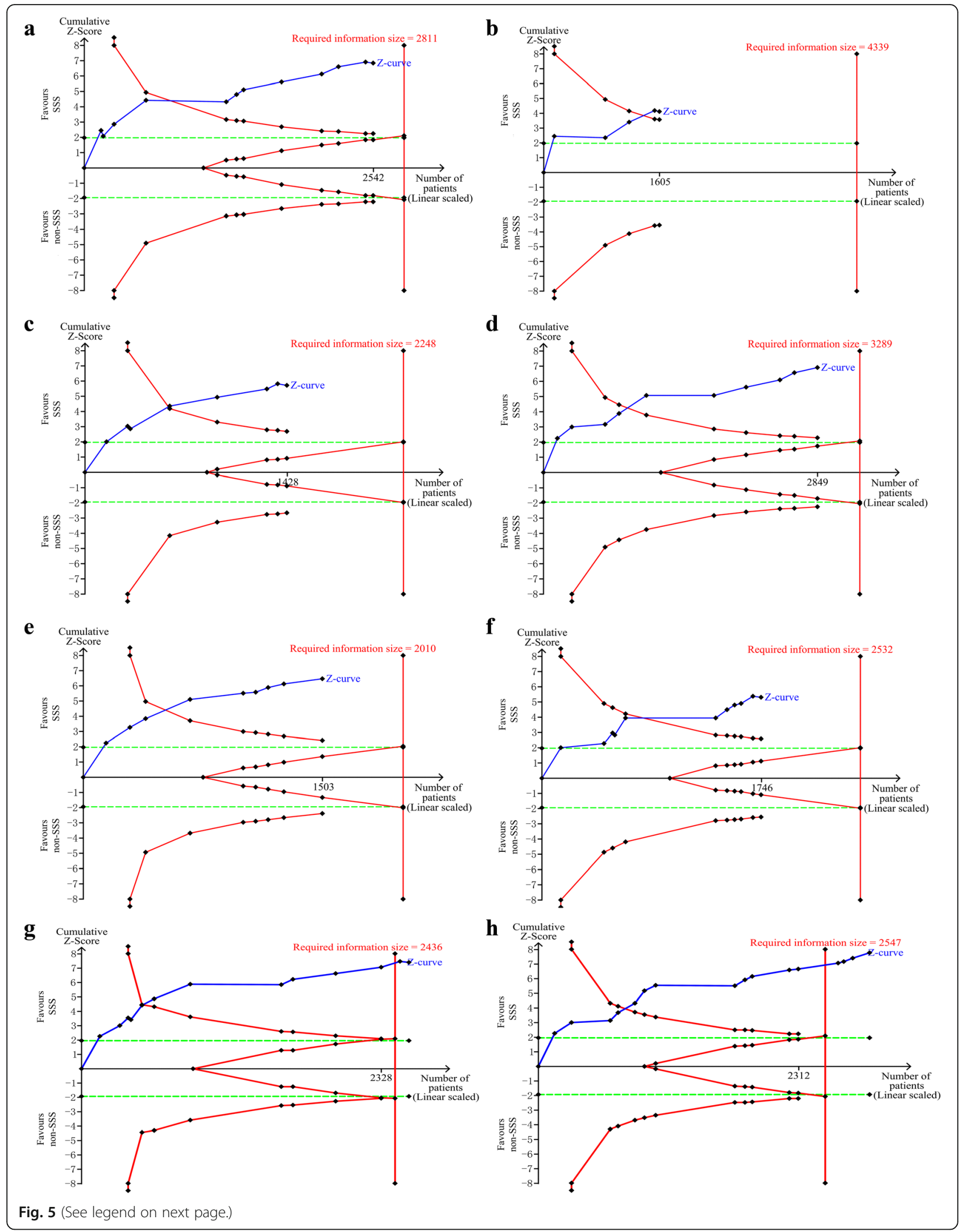


(See figure on previous page.)

Fig. 5 Trial sequential analysis for sensitivity analysis of incidence of ventilator-associated pneumonia. In (a-h) TSA plots, all Z curves have crossed the trial sequential monitoring boundary for benefit. a Trial sequential analysis in 12 appropriate randomized trials with control arm event proportion of $20.5 \%$, relative risk reduction of $20 \%$, a of $5 \%$ (two sided), $\beta$ of $20 \%$, and $l^{2}=7 \%$. The required information size was calculated as 2811 . b Trial sequential analysis in five appropriate allocation concealment trials with control arm event proportion of $14.2 \%$, relative risk reduction of $20 \%$, a of $5 \%$ (two sided), $\beta$ of $20 \%$, and $P^{2}=0 \%$. The required information size was calculated as 4339 . c Trial sequential analysis in eight appropriate assessment blinded trials with control arm event proportion of $24.5 \%$, relative risk reduction of $20 \%$, a of $5 \%$ (two sided), $\beta$ of $20 \%$, and $P=11 \%$. The required information size was calculated as 2248. d Trial sequential analysis in 10 trials including over 100 participants with control arm event proportion of $18.0 \%$, relative risk reduction of $20 \%$, a of $5 \%$ (two sided), $\beta$ of $20 \%$, and $P^{2}=0 \%$. The required information size was calculated as 3289 . e Trial sequential analysis in nine intermittent suction trials with control arm event proportion of $26.7 \%$, relative risk reduction of $20 \%$, a of $5 \%$ (two sided), $\beta$ of $20 \%$, and $P^{2}=22 \%$. The required information size was calculated as 2010. f Trial sequential analysis in 11 continuous suction trials with control arm event proportion of $22.3 \%$, relative risk reduction of $20 \%$, a of $5 \%$ (two sided), $\beta$ of $20 \%$, and $P^{2}=22 \%$. The required information size was calculated as 2532. g Trial sequential analysis in 13 trials with control arm event proportion of $23.0 \%$, relative risk reduction of $20 \%$, a of $5 \%$ (two sided), $\beta$ of $20 \%$, and $P^{2}=0 \%$. The required information size was calculated as 2436 . $\mathbf{h}$ Trial sequential analysis in 16 trials excluding multiple manipulations studies with control arm event proportion of $16.1 \%$, relative risk reduction of $20 \%$, a of $5 \%$ (two sided), $\beta$ of $20 \%$, and $P^{2}=0 \%$. The required information size was calculated as 2547 . SSS, subglottic secretion suctioning

\section{Publication bias}

For the meta-analysis of VAP including all trials no significant publication bias was observed by inspection of the funnel plot (Additional file 2: Figure S1). The Begg test and Egger test showed that no significant publication bias was detected ( $p=0.770$ and $p=0.051$, respectively).

\section{Discussion}

Our systematic review and meta-analysis found that SSS significantly reduced the incidence of VAP both in highquality trials and in all included trials. The beneficial evidence was consistent in all sensitivity analysis and was validated by TSA. Meanwhile, SSS further reduced earlyonset VAP, incidence of bacteria detection in airway secretion, duration of $\mathrm{MV}$, and delayed time-to-onset of VAP. However, SSS did not shorten length of ICU stay, or improve ICU or hospital mortality regardless of the clinical diagnosis or invasive diagnosis of VAP.

Several previous meta-analyses have reported on the same topic, as presented in Additional file 3 (Table S2) [11-16]. Differences between the present meta-analysis and the previous ones are as follows. First, our metaanalysis included three additional trials that were published recently, and these were not included in previous meta-analyses. As the latest and most comprehensively updated meta-analysis, the present study further reinforces the earlier results of previous meta-analyses. Second, we registered the protocol of this study on PROSPERO. A registered protocol may increase the transparency and quality of meta-analysis. Third, TSA was further applied to estimate the effect more conservatively. We also used TSA of subgroup analysis and sensitivity analysis to confirm the conclusion. Fourth, the present study adds outcome data to the current body of evidence as follows: SSS may prevent early-onset VAP, reduce incidence of bacteria detection, prolong time-to-onset of VAP, and reduce duration of MV. Last, but not least, we give the evidence body level using the GRADE approach. Thus, the conclusions of this study can be clinically used and transferred to guidelines.

The present meta-analysis showed that SSS may significantly reduce the incidence of VAP. We calculated the ARR (0.0953) and NNT (10.49). This value of ARR (0.0953) meant that SSS can reduce $9.53 \%$ of the absolute rate of VAP. NNT (10.49) means that for every 11 patients with SSS, one VAP was prevented. Additionally, we tried to answer a second question whether or not future research is needed to confirm this conclusion. Two current effective approaches, namely TSA and GRADE, were used.

Sensitivity analyses found that both intermittent and continuous suction can prevent VAP, with no significant difference between subgroups. It is difficult to determine which approach is appropriate because the current evidence is limited. Berra et al. reported that continuous suction was associated with widespread injury to the apptracheal mucosa/submucosa [44]. Thus, an intermittent approach may be beneficial for efficacy and safety. However, tracheal damage with subglottic secretion drainage is observed in animal studies but not in human data. Additionally, no side effects, such as tracheal damage, have been reported despite the large number of patients with SSS worldwide. Moreover, the trials [11, 13-15, 44] on SSS-related complications are rare, and further trials comparing intermittent and continuous suction are needed.

The present study suggested that SSS may significantly prevent early-onset VAP, but not late-onset VAP. The attributable mortality of late-onset VAP is higher than that of early-onset VAP, which weakens the impact of SSS on mortality from VAP. Dezfulian et al. suggested that SSS could prevent early-onset VAP in their meta-analysis, but late-onset VAP was not addressed in their study [11]. A meta-analysis by Wang et al. reported that SSS did not significantly reduce incidence of late-onset VAP based on three trials (591 patients) [15]. In the present meta-analysis, we included five trials (963 patients), and 
no significant difference was suggested. Based on the results, SSS may be suitable for patients with high risk of early-onset VAP.

Our meta-analysis also suggested that SSS can reduce incidence of bacteria detection, including both gramnegative bacteria and gram-positive bacteria causing VAP. This finding, which was not analysed in previous meta-analyses, supports the pathogenesis of VAP that leakage of fluid with bacteria passes the tracheal tube cuff toward the lungs [45-48]. Our meta-analysis also found that SSS significantly delayed the time-to-onset of VAP. According to this finding, SSS may greatly reduce incidence of VAP in patients who may have undergone early tracheal extubation. Our meta-analysis suggested that SSS can reduce the duration of MV. Interaction between the duration of MV and VAP may exist. Observational studies showed that VAP, especially early-onset VAP [49], was associated with an increased duration of MV [50]. However, most RCTs did not show a significant reduction of MV duration, whereas VAP was effectively prevented. Sample size was amplified through meta-analysis. Thus, data synthesis may introduce a different result.

This study failed to show that SSS could significantly reduce ICU and hospital mortality, as well as the length of ICU stay. There may be several reasons for this. Firstly, these outcomes may have many influencing factors or confounding bias [40, 45-48]. Secondly, VAP was associated with a $20-30 \%$ higher risk increase of mortality $[2,42]$ than for non-VAP patients; thus, an ARR (0.0953) of VAP in this study may reduce the ICU mortality by $1.9-2.9 \%$. As the mortality of the control group was $25.6 \%$ (as in our study), a very large sample size (at least 1940-4519 in each arm) would be needed to reach a significant result for ICU mortality [42]. Thirdly, the large database epidemiologic study of Rello et al. [3] also suggested that VAP-preventing strategies may not improve the survival rate, but they may provide other important advantages to patients, their families, and hospital systems.

SSS also has some disadvantages. The first disadvantage is the narrower inner lumen of the endotracheal tubes which may increase the airway resistance. Secondly, endotracheal tubes with SSS would result in unjustified incremental costs [51]. This limits the use of SSS. A prospective observational study showed that only $41.5 \%$ of all intubated patients used SSS [52].

A major strength of this study is the application of Cochrane methodology and complying with the PRISMA guidelines. Moreover, our study protocol was registered in PROSPERO. To increase the robustness of this metaanalysis, TSA was conducted to evaluate the risk of random error and repetitive testing. Sensitivity analyses based on various selection criteria all obtained a significant result both in traditional meta-analysis and in TSA, which robustly supported our primary finding. Moreover, the
GRADE approach was performed to give the level of evidence.

Our meta-analysis also has limitations. Firstly, the included RCTs in this meta-analysis were performed in different patient groups and various clinical settings. Therefore, the risk of introducing potential heterogeneity is present, although the detected heterogeneity is not significant. Secondly, because SSS is an obvious clinical manipulation, it could not be blinded for physicians and nurses; this may lead to unavoidable performance bias. Thirdly, confounding interventions, such as polyurethane, continuous control of cuff pressure, and semi-recumbent position, existed in some included trials. Therefore, we performed a sensitivity analysis excluding these trials which used other confounding interventions. Fortunately, the result of sensitivity analysis was stable. Fourthly, data on cost-effectiveness of SSS was unavailable in our metaanalysis. The main problem with SSS is the fact that, in some countries, a large number of patients are intubated before ICU admission with a tracheal tube without an SSS channel. Although previous retrospective studies suggested that SSS was cost-effective [53, 54], no RCT has conducted cost-effectiveness analysis. Thus, the widespread use of SSS is limited, and RCTs with costeffectiveness analysis may be needed.

\section{Conclusions}

This meta-analysis suggests that SSS significantly reduced the incidence of VAP, early-onset VAP, gram-positive or gram-negative bacteria causing VAP, and duration of mechanical ventilation. SSS delayed the time-to-onset of VAP. However, SSS did not show a significant difference in terms of late-onset VAP, ICU mortality, hospital mortality, ICU length of stay, incidence of tracheotomy, or reintubation. In summary, SSS is recommended to prevent VAP and to reduce ventilation length, especially in the population at high risk of early-onset VAP.

\section{Additional files}

Additional file 1: Table S1. The secondary outcomes with VAP invasive diagnosis. (DOC $56 \mathrm{~kb}$ )

Additional file 2: Figure S1. Funnel plot to evaluate potential publication bias for incidence of ventilator-associated pneumonia including all trials. $R R$ relative risk, SE standard error. (TIF $790 \mathrm{~kb}$ )

Additional file 3: Table S2. Comparison with previous meta-analyses. (DOCX $16 \mathrm{~kb})$

\section{Abbreviations}

ARR: Absolute risk reduction; Cl: Confidence interval; GRADE: Grading of Recommendations Assessment, Development, and Evaluation; ICU: Intensive care unit; MD: Mean difference; MV: Mechanical ventilation; NNT: Number needed to treat; PRISMA: Preferred Reporting Items for Systematic Reviews and Meta-Analyses; RCT: Randomized controlled trial; RR: Relative risk; SSS: Subglottic secretion suctioning; TSA: Trial sequential analysis: VAP: Ventilator-associated pneumonia 


\section{Availability of data and materials}

The authors confirm that all data underlying the findings are fully available without restriction. All relevant data are within the paper and its supporting information files.

\section{Authors' contributions}

$Z M, L G$, and GW contributed equally to this work. ZM, LG, and GW conceived the study, participated in the design, collected the data, performed statistical analyses, and drafted the manuscript. CL and WG performed statistical analyses and helped to draft the manuscript. YZ and HK collected the data and revised the manuscript critically for important intellectual content. FZ collected the data, performed statistical analyses and helped to revise the manuscript critically for important intellectual content. All authors read and approved the final manuscript.

\section{Competing interests}

The authors declare that they have no competing interests.

\section{Author details}

'Department of Critical Care Medicine, Chinese People's Liberation Army General Hospital, 28 Fu-Xing Road, Beijing 100853, People's Republic of China. ${ }^{2}$ Department of Cardiovascular Surgery, Institute of Cardiac Surgery, Chinese People's Liberation Army General Hospital, Beijing, People's Republic of China. ${ }^{3}$ Department of Orthopaedics Chinese, People's Liberation Army General Hospital, Beijing, People's Republic of China. ${ }^{4}$ Department of Anesthesiology, Drum Tower Hospital, Medical College of Nanjing University, Nanjing, China.

\section{Received: 26 July 2016 Accepted: 10 October 2016}

\section{Published online: 28 October 2016}

\section{References}

1. Vincent JL, Rello J, Marshall J, Silva E, Anzueto A, Martin CD, Moreno R, Lipman J, Gomersall C, Sakr Y, et al. International study of the prevalence and outcomes of infection in intensive care units. JAMA. 2009:302(21):2323-9.

2. Chastre J, Fagon JY. Ventilator-associated pneumonia. Am J Respir Crit Care Med. 2002;165(7):867-903.

3. Rello J, Ollendorf DA, Oster G, Vera-Llonch M, Bellm L, Redman R, Kollef MH, Group VAPOSA. Epidemiology and outcomes of ventilator-associated pneumonia in a large US database. Chest. 2002;122(6):2115-21.

4. Tablan OC, Anderson L, Besser R, Bridges C, Hajjeh R, Cdc, Healthcare Infection Control Practices Advisory C. Guidelines for preventing healthcareassociated pneumonia, 2003: recommendations of CDC and the Healthcare Infection Control Practices Advisory Committee. MMWR Recomm Rep. 2004;53(RR-3):1-36.

5. Chawla R. Epidemiology, etiology, and diagnosis of hospital-acquired pneumonia and ventilator-associated pneumonia in Asian countries. Am J Infect Control. 2008;36(4 Suppl):S93-100.

6. Collard HR, Saint S, Matthay MA. Prevention of ventilator-associated pneumonia: an evidence-based systematic review. Ann Intern Med. 2003;138(6):494-501.

7. Combes A, Luyt CE, Fagon JY, Wolff M, Trouillet JL, Chastre J. Early predictors for infection recurrence and death in patients with ventilatorassociated pneumonia. Crit Care Med. 2007;35(1):146-54

8. Dodek P, Keenan S, Cook D, Heyland D, Jacka M, Hand L, Muscedere J, Foster D, Mehta N, Hall R, et al. Evidence-based clinical practice guideline for the prevention of ventilator-associated pneumonia. Ann Intern Med. 2004;141(4):305-13.

9. Klompas M, Branson R, Eichenwald EC, Greene LR, Howell MD, Lee G, Magill SS, Maragakis LL, Priebe GP, Speck K, et al. Strategies to prevent ventilatorassociated pneumonia in acute care hospitals: 2014 update. Infect Control Hosp Epidemiol. 2014;35(8):915-36.

10. Alvarez Lerma F, Sanchez Garcia M, Lorente L, Gordo F, Anon JM, Alvarez J, Palomar M, Garcia R, Arias S, Vazquez-Calatayud M, et al. Guidelines for the prevention of ventilator-associated pneumonia and their implementation. The Spanish "Zero-VAP" bundle. Med Intensiva. 2014;38(4):226-36.

11. Dezfulian C, Shojania K, Collard HR, Kim HM, Matthay MA, Saint S. Subglottic secretion drainage for preventing ventilator-associated pneumonia: a metaanalysis. Am J Med. 2005;118(1):11-8.
12. Frost SA, Azeem A, Alexandrou E, Tam V, Murphy JK, Hunt L, O'Regan W, Hillman KM. Subglottic secretion drainage for preventing ventilator associated pneumonia: a meta-analysis. Aust Crit Care. 2013;26(4):180-8.

13. Leasure AR, Stirlen J, Lu SH. Prevention of ventilator-associated pneumonia through aspiration of subglottic secretions: a systematic review and metaanalysis. Dimens Crit Care Nurs. 2012;31(2):102-17.

14. Muscedere J, Rewa O, McKechnie K, Jiang X, Laporta D, Heyland DK Subglottic secretion drainage for the prevention of ventilator-associated pneumonia: a systematic review and meta-analysis. Crit Care Med. 2011;39(8):1985-91.

15. Wang F, Bo L, Tang L, Lou J, Wu Y, Chen F, Li J, Deng X. Subglottic secretion drainage for preventing ventilator-associated pneumonia: an updated meta-analysis of randomized controlled trials. J Trauma Acute Care Surg. 2012;72(5):1276-85

16. Caroff DA, Li L, Muscedere J, Klompas M. Subglottic secretion drainage and objective outcomes: a systematic review and meta-analysis. Crit Care Med. 2016:44(4):830-40

17. Damas P, Frippiat F, Ancion A, Canivet JL, Lambermont B, Layios N, Massion P, Morimont $\mathrm{P}$, Nys M, Piret $\mathrm{S}$, et al. Prevention of ventilator-associated pneumonia and ventilator-associated conditions: a randomized controlled trial with subglottic secretion suctioning. Crit Care Med. 2015;43(1):22-30.

18. Krein SL, Kowalski CP, Damschroder L, Forman J, Kaufman SR, Saint S. Preventing ventilator-associated pneumonia in the United States: a multicenter mixed-methods study. Infect Control Hosp Epidemiol. 2008:29(10):933-40.

19. Rello J, Lode H, Cornaglia G, Masterton R. A European care bundle for prevention of ventilator-associated pneumonia. Intensive Care Med. 2010;36(5):773-80.

20. Deem S, Yanez D, Sissons-Ross L, Elrod Broeckel JA, Daniel S, Treggiari M. Randomized pilot trial of two modified endotracheal tubes to prevent ventilator-associated pneumonia. Ann Am Thorac Soc. 2016;13(1):72-80.

21. Gopal S, Luckraz H, Giri R, Nevill A, Muhammed I, Reid M, Bickerton S, Jenkins D. Significant reduction in ventilator-associated pneumonia with the Venner-PneuX System in high-risk patients undergoing cardiac surgery: the Low Ventilator-Associated-Pneumonia study. Eur J Cardiothorac Surg. 2015;47(3):e92-96.

22. Tao Z, Zhao S, Yang G, Wang L, Zhu S. Effect of two methods of subglottic secretion drainage on the incidence of ventilator-associated pneumonia. Zhonghua Jie He He Hu Xi Za Zhi. 2014;37(4):283-6.

23. Safdari R, Yazdannik A, Abbasi S. Effect of intermittent subglottic secretion drainage on ventilator-associated pneumonia: a clinical trial. Iran J Nurs midwifery Res. 2014:19(4):376-80.

24. Koker A, Gok F, Erayman I, Yosunkaya A. Effect of subglottic secretion drainage for preventing ventilator-associated pneumonia. Crit Care. 2014;18:S119-20.

25. Moher D, Liberati A, Tetzlaff J, Altman DG, PRISMA Group. Preferred reporting items for systematic reviews and meta-analyses: the PRISMA statement. BMJ. 2009:339:b2535.

26. Higgins JPT, Green S. Cochrane Handbook for Systematic Reviews of Interventions 5.1.0. The Cochrane Collaboration. 2011. [Updated March 2011]. Available from http://handbook.cochrane.org/.

27. DerSimonian R, Laird N. Meta-analysis in clinical trials. Control Clin Trials. 1986;7(3):177-88.

28. Holst LB, Petersen MW, Haase N, Perner A, Wetterslev J. Restrictive versus liberal transfusion strategy for red blood cell transfusion: systematic review of randomised trials with meta-analysis and trial sequential analysis. BMJ. 2015;350:h1354.

29. Xia Y, Luo H, Liu JP, Gluud C. Phyllanthus species versus antiviral drugs for chronic hepatitis B virus infection. Cochrane Database Syst Rev. 2013;4:Cd009004.

30. Mahul P, Auboyer C, Jospe R, Ros A, Guerin C, el Khouri Z, Galliez M, Dumont A, Gaudin O. Prevention of nosocomial pneumonia in intubated patients: respective role of mechanical subglottic secretions drainage and stress ulcer prophylaxis. Intensive Care Med. 1992;18(1):20-5.

31. Valles J, Artigas A, Rello J, Bonsoms N, Fontanals D, Blanch L, Fernandez R, Baigorri F, Mestre J. Continuous aspiration of subglottic secretions in preventing ventilator-associated pneumonia. Ann Intern Med. 1995;122(3):179-86.

32. Kollef MH, Skubas NJ, Sundt TM. A randomized clinical trial of continuous aspiration of subglottic secretions in cardiac surgery patients. Chest. 1999:116(5):1339-46.

33. Bo H, He L, Qu J. Influence of the subglottic secretion drainage on the morbidity of ventilator associated pneumonia in mechanically ventilated patients. Zhonghua Jie He He Hu Xi Za Zhi. 2000;23(8):472-4. 
34. Smulders $\mathrm{K}$, van der Hoeven $\mathrm{H}$, Weers-Pothoff I, Vandenbroucke-Grauls C. A randomized clinical trial of intermittent subglottic secretion drainage in patients receiving mechanical ventilation. Chest. 2002;121(3):858-62.

35. Girou E, Buu-Hoi A, Stephan F, Novara A, Gutmann L, Safar M, Fagon JY. Airway colonisation in long-term mechanically ventilated patients. Effect of semi-recumbent position and continuous subglottic suctioning. Intensive Care Med. 2004;30(2):225-33.

36. Liu QH, He LX, Hu BJ, Li HY, Chen XH, Gao XD, Chu DM, Zhu HL. Comprehensive prevention and pathogenesis of ventilator-associated pneumonia in elderly patients: a prospective, randomized, case-control clinical trial. Zhonghua Nei Ke Za Zhi. 2006;45(9):717-20.

37. Liu SH, Yan XX, Cao SQ, An SC, Zhang $\amalg$. The effect of subglottic secretion drainage on prevention of ventilator-associated lower airway infection. Zhonghua Jie He He Hu Xi Za Zhi. 2006;29(1):19-22.

38. Lorente $L$, Lecuona $M$, Jimenez A, Mora ML, Sierra A. Influence of an endotracheal tube with polyurethane cuff and subglottic secretion drainage on pneumonia. Am J Respir Crit Care Med. 2007;176(11):1079-83.

39. Bouza E, Perez MJ, Munoz P, Rincon C, Barrio JM, Hortal J. Continuous aspiration of subglottic secretions in the prevention of ventilator-associated pneumonia in the postoperative period of major heart surgery. Chest. 2008;134(5):938-46.

40. Yang CS, Qiu HB, Zhu YP, Huang YZ, Xu XT, Gao L. Effect of continuous aspiration of subglottic secretions on the prevention of ventilator-associated pneumonia in mechanically ventilated patients: a prospective, randomized, controlled clinical trial. Zhonghua Nei Ke Za Zhi. 2008;47(8):625-9.

41. Zheng RQ, Lin $\mathrm{H}$, Shao J, Chen QH, Lu NF, Yu JQ. A clinical study of subglottic secretion drainage for prevention of ventilation associated pneumonia. Zhongguo Wei Zhong Bing Ji Jiu Yi Xue. 2008;20(6):338-40.

42. Lacherade JC, De Jonghe B, Guezennec P, Debbat K, Hayon J, Monsel A, Fangio P, Appere de Vecchi C, Ramaut C, Outin H, et al. Intermittent subglottic secretion drainage and ventilator-associated pneumonia: a multicenter trial. Am J Respir Crit Care Med. 2010;182(7):910-7.

43. Seyfi S, Latifi S, Shirkhani Z. Effect of subglottic secretion drainage on the ventilator-associated pneumonia in ICU patients. J Babol Univ Med Sci. 2013;15(1):58-62.

44. Berra L, De Marchi L, Panigada M, Yu ZX, Baccarelli A, Kolobow T. Evaluation of continuous aspiration of subglottic secretion in an in vivo study. Crit Care Med. 2004;32(10):2071-8.

45. Oikkonen M, Aromaa U. Leakage of fluid around low-pressure tracheal tube cuffs. Anaesthesia. 1997;52(6):567-9.

46. Pavlin EG, VanNimwegan D, Hornbein TF. Failure of a high-compliance low-pressure cuff to prevent aspiration. Anesthesiology. 1975;42(2):216-9.

47. Petring OU, Adelhoj B, Jensen BN, Pedersen NO, Lomholt N. Prevention of silent aspiration due to leaks around cuffs of endotracheal tubes. Anesth Analg. 1986;65(7):777-80.

48. Young PJ, Rollinson M, Downward G, Henderson S. Leakage of fluid past the tracheal tube cuff in a benchtop model. Br J Anaesth. 1997;78(5):557-62.

49. Li S, Zhang Y, Li S, Wang X, Zhang R, Lu Z, Yan J. Risk factors associated with prolonged mechanical ventilation after corrective surgery for Tetralogy of Fallot. Congenit Heart Dis. 2015;10(3):254-62.

50. Zhu S, Cai L, Ma C, Zeng H, Guo H, Mao X, Zeng C, Li X, Zhao H, Liu Y, et al. The clinical impact of ventilator-associated events: a prospective multi-center surveillance study. Infect Control Hosp Epidemiol. 2015;36(12):1388-95.

51. Loupec T, Petitpas F, Kalfon P, Mimoz O. Subglottic secretion drainage in prevention of ventilator-associated pneumonia: mind the gap between studies and reality. Crit Care. 2013;17(6):R286.

52. Lorente L, Lecuona M, Jimenez A, Lorenzo L, Roca I, Cabrera J, Llanos C, Mora ML. Continuous endotracheal tube cuff pressure control system protects against ventilator-associated pneumonia. Crit Care. 2014;18(2):R77.

53. Speroni KG, Lucas J, Dugan L, O'Meara-Lett M, Putman M, Daniel M, Atherton M. Comparative effectiveness of standard endotracheal tubes vs. endotracheal tubes with continuous subglottic suctioning on ventilatorassociated pneumonia rates. Nurs Econ. 2011;29(1):15-20. 37.

54. Shorr AF, O'Malley PG. Continuous subglottic suctioning for the prevention of ventilator-associated pneumonia: potential economic implications. Chest. 2001:119(1):228-35.

\section{Submit your next manuscript to BioMed Central and we will help you at every step:}

- We accept pre-submission inquiries

- Our selector tool helps you to find the most relevant journal

- We provide round the clock customer support

- Convenient online submission

- Thorough peer review

- Inclusion in PubMed and all major indexing services

- Maximum visibility for your research

Submit your manuscript at www.biomedcentral.com/submit

) Biomed Central 